\title{
Evaluation of surface profile of holographic diffraction reflective coatings on scattering chart using in laser alarm systems
}

\author{
SergeyHerasimov ${ }^{1}$,Yaroslav Kozhushko ${ }^{2}$, EvgeniyRoshchupkin ${ }^{3}$, Volodymyr Dekadin ${ }^{4}$, \\ Volodymyr Djus ${ }^{5}$,Yevgen Melenti ${ }^{6}$ \\ ${ }^{1}$ Ivan KozhedubKharkiv National Air Force University, Kharkiv, Ukraine, gsvnr@ukr.net \\ ${ }^{2}$ Ivan KozhedubKharkiv National Air Force University, Kharkiv, Ukraine, oficer2003@ukr.net \\ ${ }^{3}$ Ivan KozhedubKharkiv National Air Force University, Kharkiv, Ukraine, reszzz76@ gmail.com \\ ${ }^{4}$ Ivan KozhedubKharkiv National Air Force University, Kharkiv, Ukraine, decadin75@gmail.com \\ ${ }^{5}$ Ivan KozhedubKharkiv National Air Force University, Kharkiv, Ukraine, vdjus@ukr.net \\ ${ }^{6}$ Juridical Personnel Training Institute for the Security Service of Ukraine YaroslavMudryi National Law \\ University, Kharkiv, Ukraine, melenty@ukr.net
}

\begin{abstract}
The evaluation analysis dependingon the type of holographic diffraction diagram scattering reflective coating on surface profile mentioned period is carried out. Change tendencies of charts type diffractive scattering reflective coatings period of growth surface profile are found out. The prospects of the use of holographic diffraction reflective coating composed of laser alarm systems. Determined that the value of the value of the period of the surface profile of holographic diffraction reflective coating significantly affects the type of scattering diagram of these coatings. Diagrams of dispersion diffraction reflective coating in relative units are given.
\end{abstract}

Key words :diffraction reflective coating, dispersion pattern, laser alarm systems, scattering chart.

\section{INTRODUCTION}

Current development trends and the widespread use of alarm systems are important part of the automated protection complex (security) of objects and approaches to them, necessitating the development of new and improvement of existing systems. This issue is especially relevant when solving the problem of increasing the level of protection of such objects from terrorist attacks and sabotage[1].In this respect laser alarm systems become actual for the following reasons[2] - [12]:

contactless systems;

low weight and size parameters;

high noise immunity noiseless;

small power consumption;

easy installation, preparation and adjustment.
Among the disadvantages of laser alarm systems it is necessary to note the presence of false positives under adverse conditions, reducing the environment transparency where laser radiation distributed. Removing this limitation disadvantage is ensured by multiple laser energy excess over the minimum threshold, necessary for the system operation, as and one of perspective solutions is the formation of a certain number of "laser barriers" in different directions.

\section{LITERATURE REVIEW}

Technical realization of laser alarm systems provides the location of a laser transmitter and a photodetector at one track end of the laser beam spread, and the other one is light reflectors (in the simplest case it is mirror). In turn, the use of diffraction reflective coatings (DRCs) as the reflective element within the system allows you to focus a significant part of the energy of the reflected laser light in one or more narrow angular sectors. So is the formation of one or more "laser barriers". This fact is due to the fact that the DRCs are engaged in the energy redistribution of reflected laser light in space. Thus, there is a transition from uniform reflectance, which is described by Lambert, to significantly uneven distribution characteristic for the laser reflection at diffraction grating [2],[3], [13],[14].

DRCs can be characterized by different profiles and surface parameters (period, height profile). Various DRCs have significantly different scattering charts, which leads to the need to develop guidance on the use of specific types of DRCs composed of laser alarm systems intended for use in fixed objects[3], [15]-[21].

Holographic DRCs surface profile function is a harmonic function - cosine, technically just sell and its production does not require significant investment. For these reasons, holographic coverage of particular interest for their use in laser alarm systems[2], [16],[22]-[28]. 
The purpose of the work is to assess the period impact of the surface profile of holographic DRCs on the chart for recommendations on the use of such DRCs consisting of laser alarm systems.

\section{MAIN MATERIAL}

To achieve this target, we consider the calculation method of DRCs scattering charts.

Currently theories of solution scattering of waves in periodic structures (diffraction gratings) are developed sufficiently. The most common and well-proven experimental research in the field are two methods:

the method of small perturbations;

Kirchhoff method.

The method of small perturbations considers optical radiation as adjustable electromagnetic field and based on accurate boundary conditions on the surface, so adequately describes the polarizing effects. However, its use is advisable in cases where the profile height of the periodic structure is quite small compared to the wavelength of the incident light and the parameters of the optical field (polarization) are known. Therefore the use of this method to determinate DRCs radiation scattering chart is not appropriate.

Use of Kirchhoff method provides a relatively simple task decision of reflection (diffraction) waves on the diffraction grating, whose results are confirmed by research method and can be directly applied in practice. This method can be applied to surfaces with a radius of curvature greater than the wavelength of incident optical radiation, also restrictions don't impose on the irregularities inclinations with respect to the surface area and polarization of optical radiation. Solution of the problem based on Fresnel-Kirchhoff diffraction equation field, which involves finding reflected from the diffractive optical element of the field by Fourier expression for the complex amplitude of the field on the surface of DRCs. Thus, during the assessment Kirchhoff method will be applied, but taking into account the profile frequency of holographic coating cosine structure.

We accept that the laser wave with $\lambda$ length extends towards $\mathrm{i}_{1}=\left\{\cos \alpha_{1}, \cos \beta_{1},-\cos \gamma_{1}\right\} \quad$ (Figure 1). Define the observation point $\left(\cos \alpha_{2}, \cos \beta_{2}, \cos \gamma_{2}, R\right)$, which is

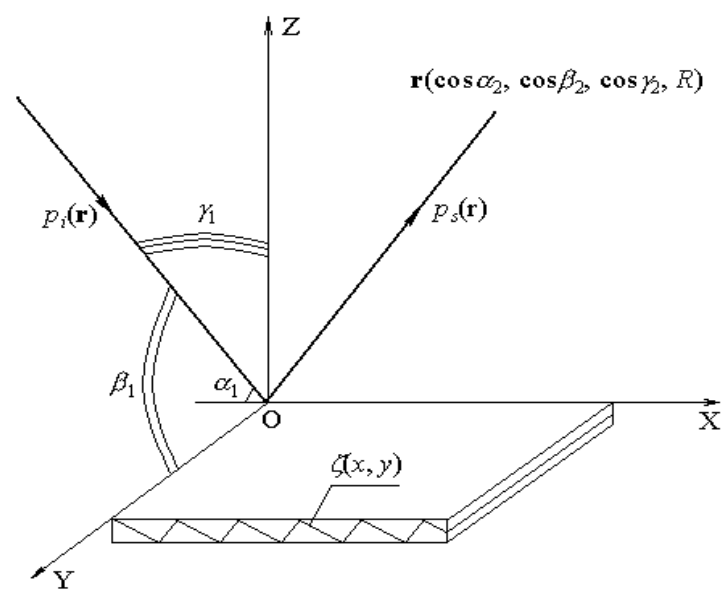

Figure 1: Cyrillic drop of laser at reflective relief phase of DRCs located at a distance $\mathrm{R}$ surface coating that is highlighted.

In this case, the calculating of one-dimensional DRCs scattering diagram looks like [3],[14]:

$$
\begin{aligned}
& \mathrm{P}_{\mathrm{V}}\left(\theta_{\mathrm{i}}, \theta_{\mathrm{S}}\right)=\mathrm{R}_{\mathrm{f}}\left(\cos \gamma_{1}+\cos \gamma_{2}\right) \times \\
& \times\left\{\mathrm{ik}\left[\begin{array}{l}
\mathrm{x}\left(\cos \alpha_{1}-\cos \alpha_{2}\right)-\mathrm{ik} 2 \zeta(\mathrm{x}) \cos \gamma_{1}+ \\
+\mathrm{y}\left(\cos \beta_{1}-\cos \beta_{2}\right)
\end{array}\right]\right\} \mathrm{ds},
\end{aligned}
$$

where $R_{f}$ is reflection coefficient of coverage;

$$
\begin{aligned}
& \mathrm{z}=\zeta(\mathrm{x}) \text { is function of profile surface coating height; } \\
& \mathrm{k}=\frac{2 \pi}{\lambda} \text { is wave number. }
\end{aligned}
$$

Integral to (1) sets at the area $\mathrm{S}$, which is illuminated by laser radiation.

Define a plane OXY length of the highlighted DWC, on the $\mathrm{OX}$ axis is $\mathrm{D}_{1}$, and along the axis $\mathrm{OY}-\mathrm{D}_{2}$. Then for flat one-dimensional DRCs expression for the back scatter chart is:

$$
\begin{aligned}
& \mathrm{P}_{\mathrm{V}}\left(\theta_{\mathrm{i}}, \theta_{\mathrm{s}}\right)=\mathrm{R}_{\mathrm{f}}\left(\cos \gamma_{1}+\cos \gamma_{2}\right) \times \\
& \times \int_{-\frac{\mathrm{D}_{2}}{2}}^{\frac{\mathrm{D}_{2}}{2}} \exp \left\{\mathrm{iky}\left(\cos \beta_{1}-\cos \beta_{2}\right)\right\} \mathrm{dy} \times \\
& \times \int_{-\frac{\mathrm{D}_{1}}{2}}^{\frac{\mathrm{D}_{1}}{2}} \exp \left\{\mathrm{ikx}\left(\cos \alpha_{1}-\cos \alpha_{2}\right)-\mathrm{ik} 2 \zeta(\mathrm{x}) \cos \gamma_{1}\right\} \mathrm{dx}
\end{aligned}
$$

In consideration of the frequency of DRC structure (phase diffraction grating) and the assumption that is on the aperture DRCs area, highlighted by laser radiation, $\mathrm{S}$ fits a whole number of periods diffraction grating on the axis OX (ie in the plane OXY length of the highlighted is $\mathrm{D}_{1}[-(\mathrm{M}+1) \mathrm{d} / 2,(\mathrm{M}+1) \mathrm{d} / 2]$ and aperture includes $\mathrm{M}+1$ grating period along the axis OX), expression (2) is reduced for appearance:

$$
\begin{gathered}
P_{\mathrm{V}}\left(\theta_{\mathrm{i}}, \theta_{\mathrm{S}}\right)=\mathrm{R}_{\mathrm{f}}\left(\cos \gamma_{1}+\cos \gamma_{2}\right) \times \\
\times \int_{-\frac{\mathrm{D}_{2}}{2}}^{\frac{\mathrm{D}_{2}}{2}} \exp \left\{\mathrm{iky}\left(\cos \beta_{1}-\cos \beta_{2}\right)\right\} \mathrm{dy} \times \\
\times \sum_{\mathrm{j}=-\mathrm{M}}^{\mathrm{M}} \int_{-\frac{\mathrm{d}_{1}}{2}}^{\frac{\mathrm{d}_{1}}{2}} \exp \left\{\mathrm{ikx}\left(\cos \alpha_{1}-\cos \alpha_{2}\right)-\mathrm{ik} 2 \zeta(\mathrm{x}) \cos \gamma_{1}\right\} \mathrm{dx} .
\end{gathered}
$$

The total number of orders that apply in the case of arbitrary 
radiation falling on DRCs (diffraction grating) is $\left(\frac{2 d}{\lambda} \sin \beta_{1}+1\right)[6]$. In the case of radiation fall back touches of DRCs at $\left(\beta_{1}=\frac{\pi}{2}\right)$ (Figure 2), the numberof ordersdegrees will be maximized for a given period of phase structure.

The expression to calculate back coverage of scatter chart is simplified due to falling of optical radiation perpendicularly to stroke of DRCs $\left(\beta_{1}=\frac{\pi}{2}\right)$ and falling areas matching and surveillance laser radiation. For this condition in [8] the following expression for the chart of dispersion coating is obtained:

$$
\begin{aligned}
& \mathrm{P}_{\mathrm{V}}\left(\theta_{\mathrm{i}}, \theta_{\mathrm{s}}\right)=\mathrm{R}_{\mathrm{f}}\left(\cos \theta_{\mathrm{i}}+\cos \theta_{\mathrm{s}}\right) \times \\
& \times \frac{\sin \left\{\frac{\mathrm{M}+1}{2} \mathrm{kd}\left(\sin \theta_{\mathrm{s}}-\sin \theta_{\mathrm{i}}\right)\right\}}{\sin \left\{\frac{\mathrm{kd}}{2}\left(\sin \theta_{\mathrm{s}}-\sin \theta_{\mathrm{i}}\right)\right\}} \times, \\
& \times \int_{-\frac{\mathrm{d}_{1}}{2}}^{\frac{\mathrm{d}_{1}}{2}} \exp \left\{\mathrm{ik}\left(\mathrm{x} \sin \theta_{\mathrm{i}}-2 \zeta(\mathrm{x}) \cos \theta_{\mathrm{i}}\right)\right\} \mathrm{dx} .
\end{aligned}
$$

when $\theta_{\mathrm{s}}, \theta_{\mathrm{i}}$ are angles, that define the position of observation points and DRCs highlighting (Figure 2).

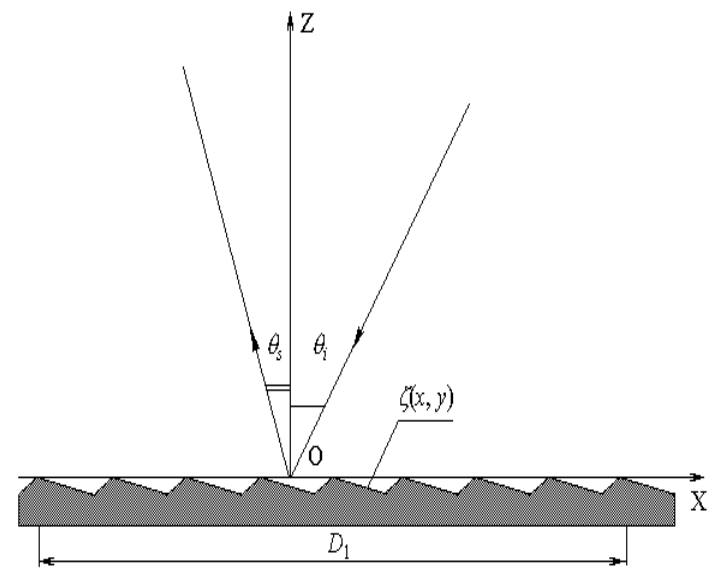

Figure 2: Fall laser perpendicular touches of phase DRCs

Expressions (3) and (4) allow to calculate the intensity of the reflected laser from DRCs for various profiles surface coating. This is possible because the intensity of the light emission is proportional to the square of the complex amplitude of the optical field [2]:

$$
\mathrm{I}_{\mathrm{H}}\left(\theta_{\mathrm{i}}, \theta_{\mathrm{s}}\right)=\mathrm{P}_{\mathrm{V}}\left(\theta_{\mathrm{i}}, \theta_{\mathrm{s}}\right) \mathrm{P}_{\mathrm{V}} *\left(\theta_{\mathrm{i}}, \theta_{\mathrm{s}}\right) \text {. }
$$

Based on relations (4) and (5) in the case of normal incidence of laser radiation on the surface of the coating in MATHCAD mathematical package conduct calculation of normalized diagram of the spatial intensity distribution of radiation scattered perfectly reflective holographic DRCs, the function of the surface profile of which is a harmonic function cosine (Figure3).

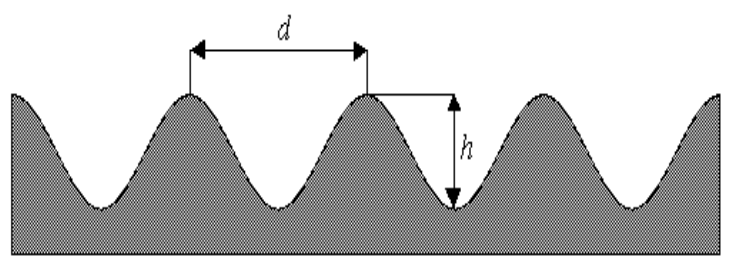

Figure 3: Profile holographic DWC

Holographic DRCs of particular interest to solve the problem of security because their production has learned.

The calculation results of holographic DRCs scattering charts at different parameters are shown in Figure4.

Analysis of calculated holographic DRCs scattering diagrams with different period d, allow to make the following conclusions:

with the growth of $d$ period from $1 \lambda$ to $5 \lambda$ increasing number of diffraction maximums scattering diagrams - areas in which the spread of the reflected laser DRCs: from 3 to 11;

varying the period $\mathrm{d}$ from $1 \lambda$ to $5 \lambda$ lead to changing the provisions of the diffraction maximums scattering diagrams areas in which the spread on the reflected from DRCs laserradiation;

the intensity of the reflected optical radiation diffraction maxima is different, that indicate about the uneven energy of reflected laser radiation in each direction;

obtained calculations results of holographic DRCs scattering diagram, are analogous to holographic DRC, obtained experimentally [5].

Thus, depending on the type of object, his configuration, it is possible to use holographic DWC with specially calculated or determined parameters.

For example, a laser alarm systems used for security in the narrow areas with significant length (corridors, tunnels) appropriate use of holographic DRCs period $\mathrm{d}=(1 \ldots 2) \lambda$, which will ensure the formation 3...5 "laser barriers".

Thus, in the central angular sector $100^{\circ}$ formed $1 . . .3$ "laser barrier".

In turn, the use of holographic DRCs with period $d=(3 \ldots .5) \lambda$ is expedient in parts of laser systems, that are quite in a wide premises and open space, the location of possible on angles in controlled areas. These holographic DRCs in angular sector $100^{0}$ will provide forming 5...9 "laser barriers".

This number of "laser barriers" also allows in addition use laser alarm system:

identifying directions of objects in different directions; measurement of the speed of objects movement. 


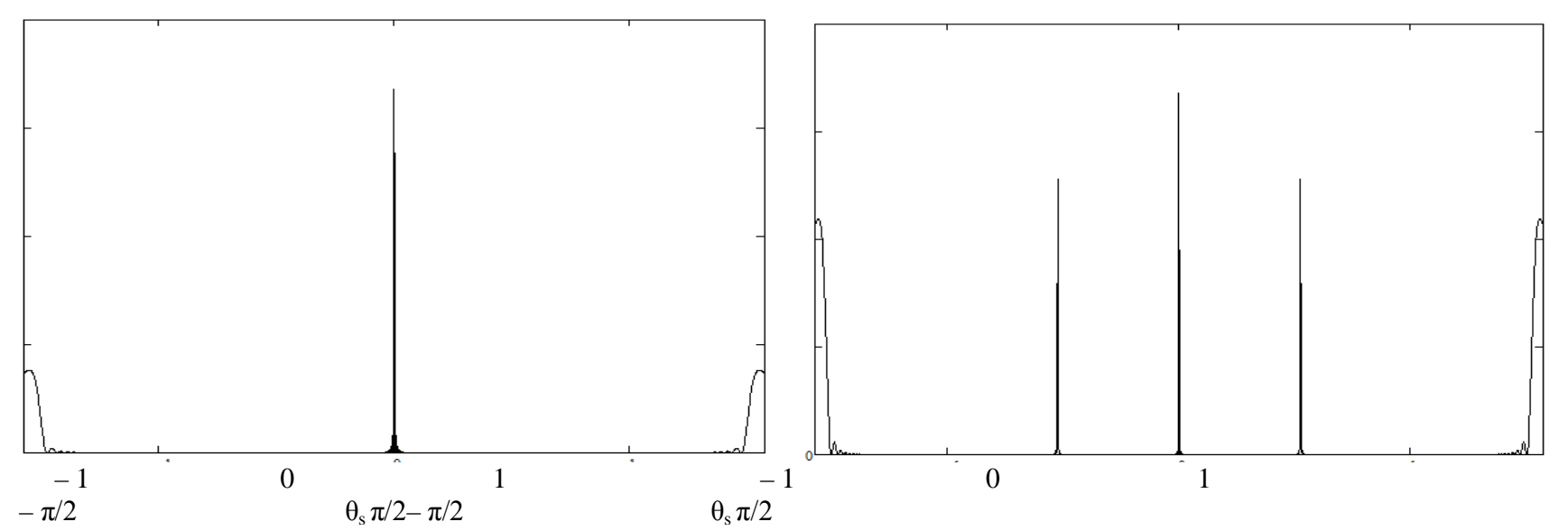
a)d=1 $\lambda, M=100$
b)d $=2 \lambda, M=100$
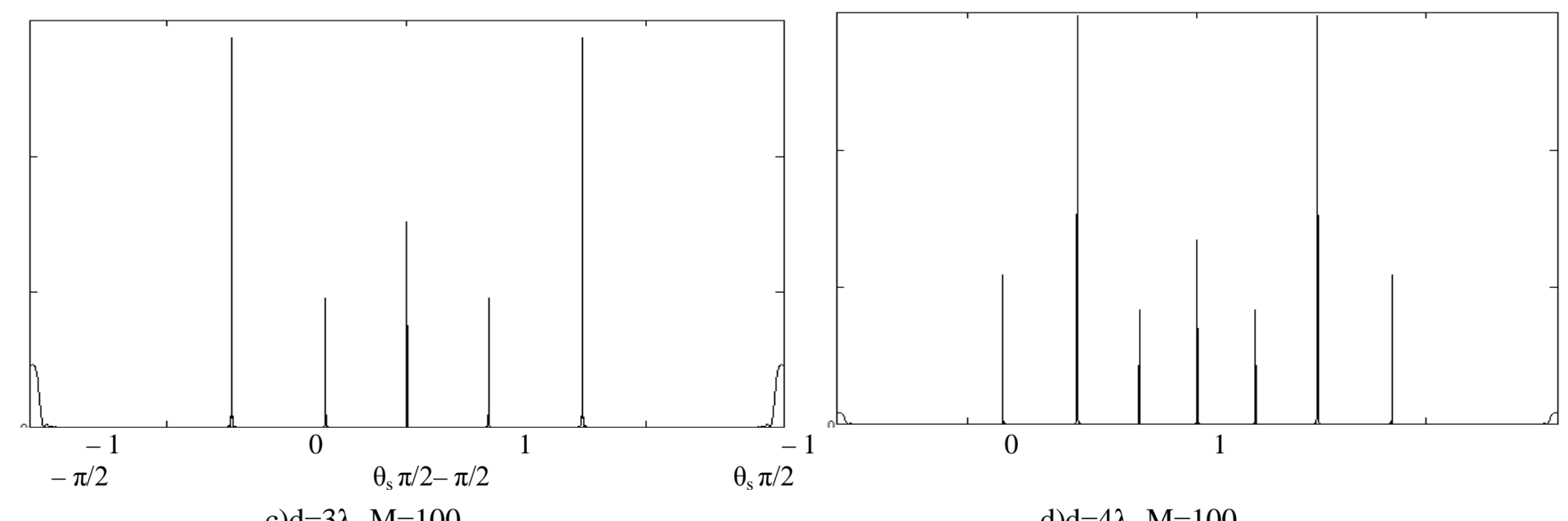

c) $\mathrm{d}=3 \lambda, \mathrm{M}=100$

d)d $=4 \lambda, M=100$

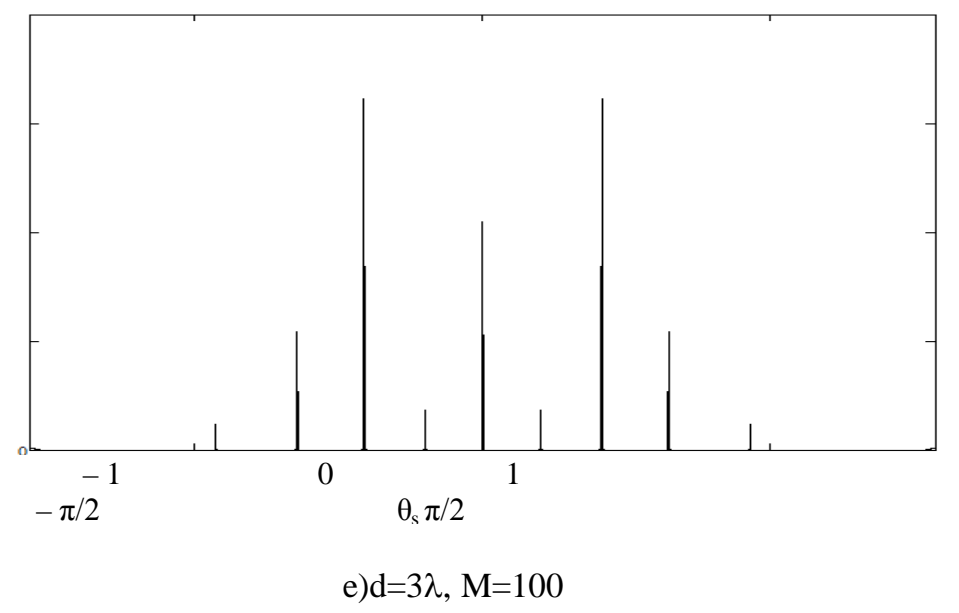

Figure 4: Chart holographic scattering DWC in relative units 


\section{RESULTS AND DISCUSSIONS}

The article shows that the power of the input signal depends on the transparency of the atmosphere and can vary significantly in accordance with weather conditions. The developed scheme allows to isolate the useful component in a wide range of input signal power, even when the receiving part is illuminated by powerful light sources (searchlight or solar radiation). This ensures high noise immunity of laser security systems.

The article developed proposals on the possibilities of expanding the list of security functions in laser signaling systems, namely, the implementation of the following functions: measuring the positions of objects (violators) and their movements at different distances; measuring the speed of movement of objects (violators); measurement (control) of the dimensions of objects (violators).

Improvement of laser alarm systems in order to expand the list of security functions is relevant for the effective protection of objects.

The work demonstrates the feasibility of holographic DWC using, whose production is technically just realized, in the laser alarm systems to improve the efficiency of these systems. Determined, that the value of the period of holographic DRCs profile surfacesignificantly affects the type of diagrams dispersion these coatings.

Based on the holographic DRCs scattering diagrams established upward trends in the number of diffraction maximums scattering diagrams - directions in which the spread the reflected laser from DRCs and redistribution of energy reflected radiation in these directions. Thus, with increasing quantities period surface profile $\mathrm{d}$ from $1 \lambda$ to $5 \lambda$, increasing the number of diffraction maximums diagrams scattering from 3 to 11 . Thus, the growth period $\mathrm{d}$ by the amount of radiation wavelength leads to two additional diffraction maxima. This effect increases the sensitivity of laser alarm systems.

\section{CONCLUSIONS}

The study of issues of effective ensuring the safety of the population and industrial facilities in modern conditions is especially relevant in connection with the intensification of threats of international terrorism and man-made disasters.

The article evaluates the period of impact of the surface profile of holographic DRCs on the chart for recommendations on the use of such DRCs consisting of laser alarm systems.

This allows to form recommendations for selection of parameters for DRCs namely the period of the surface profile, as a part of laser systems for signaling different objects of complex configuration.

Further research on the possibilities of using holographic DRCs in laser alarm systems should be directed to determine the effect of errors in manufacturing coating to characteristics DRCs.

\section{ACKNOWLEDGEMENT}

This work was supported by the Ivan KozhedubKharkiv National Air Force University(Kharkiv, Ukraine).

\section{REFERENCES}

1. Ye.Melenti, Building a threat model to assess the vulnerability of critical infrastructure, Strategic anti-crisis management: global challenges and role of the state: International scientific and practical conference, Venice, 2019, p.p. 85-89.

2. A. Katunin, R. Sidorenko, Y. Kozhushko, and G. Rybalka, Expansion of security functions of laser alarm systems, Information processing systems, № 2(100), 2012, p.p. 54-57.

3. S. Herasimov, O. Tymochko, O. Kolomiitsev, G. Aloshin, O. Kriukov, O. Morozov, and V. Aleksiyev, Formation Analysis Of Multi-Frequency Signals Of Laser Information Measuring System, EUREKA: Physics and Engineering, vol. 5, 2019, p.p. 19-28, https://doi.org/10.21303/2461-4262.2019.00984.

4. A. Katunin, V. Bzot, O. Kolodiy, and O. Lukovsky, The use of radiant infrared systems to protect the perimeters of objects in difficult weather conditions, Weapons systems and military equipment, № 2(22), 2010, p.p. 192-195.

5. S. Herasimov, E. Roshchupkin, V. Kutsenko, S. Riazantsev, and Yu. Nastishin, Statistical analysis of harmonic signals for testing of Electronic Devices, International Journal of Emerging Trends in Engineering Research, vol.8,is. 7, 2020, p.p. 3791-3798,https://doi.org/10.30534/ijeter/2020/1438720 20.

6. P. Ayswarya, S. Pournami, and Ravi Nambiar, A Survey on Ring Laser Gyroscope Technology, International Journal of Computer Applications, vol. 116, is. 2, 2015, p.p. 25-27.

7.Y. Kozhushko, Improvement of laser alarm systems based on the use of diffraction reflective coatings, Weapons systems and military equipment, № 2(42), 2015, p.p. 106-108.

8. G. Dolya, and A. Katunin, On the possibility of reducing the visibility of targets for protection against high-precision weapons on the basis of the use of diffraction-reflecting coatings, Collection of scientific works, № 2(18), 2000, p.p. 75-81.

9. S. Herasimov, V. Pavlii, O. Tymoshchuk, M.Yu. Yakovlev, D.Ye. Khaustov, Ye. Ryzhov, L. Sakovych, and Yu.A. Nastishin, Testing Signals for Electronics: Criteria for Synthesis, Journal of Electronic Testing, vol. $35, \quad$ is. 148,2019 , p.p. 1-9, https://doi.org/10.1007/s10836-019-05798-9.

10. A. Goncharsky, V. Popov, and V. Stepanov, Introduction to Computer Optics, Moscow, MGU, 163 p., 1991.

11. N. Vukainovi, and J. Duhovnik,Optical 3D Geometry Measurments Based on Laser Triangulation, Advanced CAD Modeling, 2018, p.p. 191-216, https://doi.org/10.1007/978-3-030-02399-7_9.

12. S. Herasimov, Y. Belevshchuk, I. Ryapolov, O. Tymochko, M. Pavlenko, O. Dmitriiev, 
M. Zhyvytskyi, and N. Goncharenko, Characteristics of radiolocation scattering of the SU-25T attack aircraft model at different wavelength ranges, Information and controlling systems, Eastern-European Journal of Enterprise Technologies, № 6/9 (96), 2018, p.p. 22-29, https://doi.org/10.15587/1729-4061.2018.152740.

13. Shin Kihong, On the Selection of Sensor Locations for the Fictitious FRF based Fault Detection Method, International Journal of Emerging Trends in Engineering Research, vol. 7, is. 7, 2019, p.p. 569-575, https://doi.org/10.30534/ijeter/2019/277112019.

14. Y. Kozhushko, D. Karlov, O. Klimishen, M. Bortsova, S. Herasimov, O. Hrichanuk, and V. Bykov, Comparison of the Efficiency of Some Images Superposition Algorithms Used in Aircraft Map-Matching Navigation Systems, 2018 IEEE International Conference on Mathematical Methods in Electromagnetic Theory, 2018, p.p. 282-285.

15. O. Katz, and I. Spevak, Diffraction of electromagnetic waves, Kharkiv, KhVU, 178 p., 1998.

16. O.S. Makarenko, D.A. Krushinskii, and O.S. Makarenko, Moddeling of pedestrians movement on the base of cellular automata, System research and information technologies, № 1, 2010, p.p. 100-109.

17. M.A. Facas Vicente, Design, Modelation and Numerical Simulation of a Novel Artefact for Coordinate Measuring Machines Calibration Based on Laser Trilateration), Arabian Journal for Science and Engineering, vol. 38, is.6,2013, p.p. 1519-1528, https://doi.org/10.1007/s13369-013-0537-x.

18. Hong-e Ren,and Wei Yan, The Test Principle and Algorithm of Laser Measuring Tree Height, Informatics in Control, Automation and Robotics, 2012, p.p. $\quad 381-387$, https://doi.org/10.1007/978-3-642-25899-2_51.

19.S.Menzel, L.Diehl, and C. Pflügel,Quantum cascade laser master-oscillator power-amplifier with $1,5 \mathrm{~W}$ output power at 300 K, Optics Express, vol. 19, is. 17, 2011, p.p. 16229-16235.

20.W.H.Cheng, M.T.Sheen, G.L.Wang, S.C.Wang, andJ.H. Kuang,Fiber alignment shift formation mechanisms of fiber-solder-ferrule joints in laser module packaging,Technol, vol. 19, is. 8, 2001, p.p. $1177-1184$.

21. Y.Kozhushko, D.Kolomiets, I.Tabakova, I. Hannoshyna, N. Serdiuk, andT.Voichenko, Determination of accuracy of low-altitude navigation of dynamic objects equipped with correlation-extreme systems, International Journal of Advanced Trends in Computer Science and Engineering, vol.9, is.3, 2020, p.p. 2981-2986, https://doi.org/10.30534/ijatcse/2020/75932020.

22. Y.Lin, C.Eichele, and F.G.Shi. Effect of Welding Sequence on WeldingInduced-Alignment-Distortion in Packaging of Butterfly Laser Diode Modules,Simulation and Experiment,Technol, vol. 23, is. 2, 2005, p.p. 615-623.

23. W.Bewley, C.Canedy, andC.Kim,Continous-wave interband cascade lasers operating above room temperature at $\lambda=\mathbf{4 , 7 - 5 , 6} \boldsymbol{\mu \mathrm { m }}$, Optics Express, vol. 20, is. 3, 2012, p.p. $3235-3240$.
24. High Brightness Diode Laser Modules, The catalogue of the company JENOPTIK Germany GmbH, Diode Laser Group, Germany, 2012, available at: www.jenoptik-com/en-semiconductor-lasers.

25.Laser modules, diode line and a solid-state diode-pumped lasers, Catalogue of CJSC "Semiconductor devices", Saint-Petersburg, 2016, available at: www.atcsd.ru/prodl.php.

26. Diode-Pumped Q-switched Laser Systems, Prospect firm General Atomics Photonics Division, USA, 2005.

27. A.F. Maslov, E.S. Roshchupkin, S.I. Khmelevskyi, andV.N. Selevko, Potential accuracy of measuring of the delay time by taking into account the phase structure of the signals received by the spaced apertures, Proceedings, N. 3 (41), 2002, 83-85.

28. S. Gerasimov, S. Kukobko, Y.Roshchupkin, andO. Rasstrigin,Synthesis of measurement signals for determination of technical state of automatic control systems, Weapons and military Equipment, № 4 (12), 2016, p.p. 32-36, http://www.irbis-nbuv.gov.ua/cgi-bin/irbis_nbuv/cgiirbi s_64.exe?C21COM=2\&I21DBN=UJRN\&P21DBN=UJ RN\&Z21ID=\&IMAGE_FILE_DOWNLOAD=1\&Imag e_file_name=PDF\%2Fovt_2016_4.pdf. 